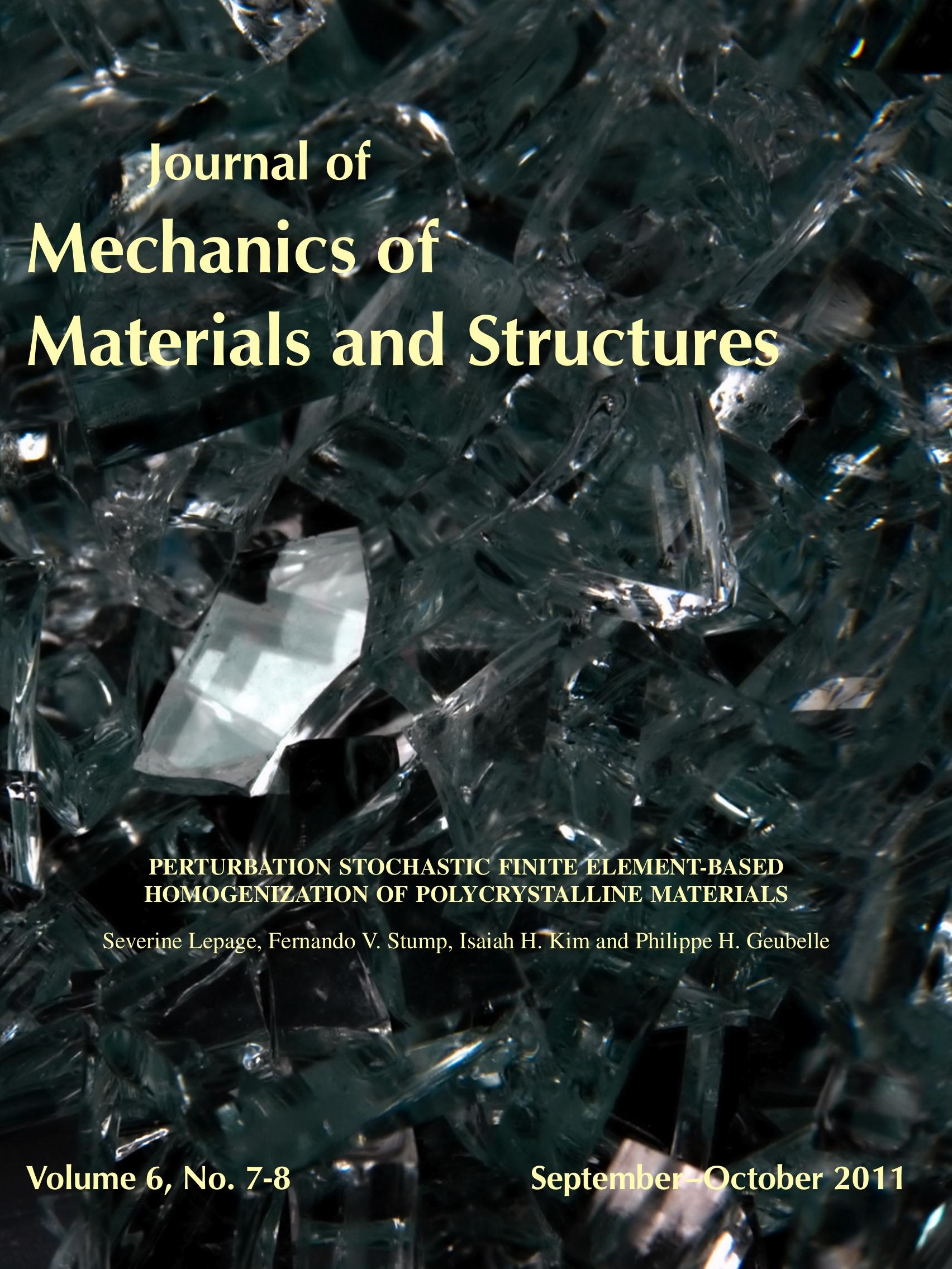




\title{
PERTURBATION STOCHASTIC FINITE ELEMENT-BASED HOMOGENIZATION OF POLYCRYSTALLINE MATERIALS
}

\author{
Severine Lepage, Fernando V. Stump, Isaiah H. Kim and Philippe H. Geubelle
}

This work presents a study of the influence on the macroscopic (homogenized) elastic properties of polycrystalline materials induced by uncertainties in the material texture and microstructure geometry. Since many microelectromechanical systems are made of materials deposited as thin films with $\langle 111\rangle$ fiber texture, we study the variance of the homogenized elastic properties of the material around its nominal $\langle 111\rangle$ texture. To perform this analysis, the perturbation stochastic finite element method (PSFEM) is coupled to the mathematical theory of homogenization leading to a second-order perturbation-based homogenization method. This method is able to evaluate the mean and variance of a given homogenized property as a function of the grain property uncertainty. The multiscale formulation is implemented in a plane-stress linear elastic finite element framework based on a multigrain periodic unit cell generated by Voronoi tessellation. This perturbation-based homogenization method is verified against Monte Carlo simulations, showing its effectiveness and limitations. Then, through applications, it is evaluated how different levels of uncertainty in grains induce uncertainty in the macroscopic elastic properties of the polycrystalline material. In particular, the influence of the unit cell is studied. Finally, by coupling the PSFEM with the Monte Carlo method, the effects on the macroscopic properties of uncertainty of both the geometry and orientation of the grains is estimated.

\section{Introduction}

The performance of a microelectromechanical system (MEMS) is affected by uncertainties. Manufacturing processes may leave substantial uncertainties in the shape and geometry of the device, while the material properties of a component are inherently subject to scattering. Moreover, microscale properties are extremely sensitive to process variations. Accuracy and precision of standard processes are difficult to control, so properties vary by recipe, by fabrication run, wafer-to-wafer, and across the wafer. The design of accurate MEMS has to take into account the influence of these uncertainties. This paper focuses on the prediction of material property variability that is inherent in MEMS due to their microfabrication processes. The materials making up MEMS are deposited as thin films. In particular, this work is motivated by the study of texturized gold thin films which are common MEMS materials. Although the electric characterization of metallic thin films is well established, the mechanical characterization of the same films is still a challenge. Moreover, the material properties are dependent on the fabrication process and therefore are not the same as those for the bulk material. The material properties can even be different between runs of the same fabrication process.

One approach to investigating property variability of metallic thin films is to use homogenization methods that allow the evaluation of the macroscopic properties based on the polycrystalline microstructure.

Keywords: perturbation stochastic finite element, homogenization, Monte Carlo method, polycrystalline material. 
By that approach the microscopic features, which are defined by the microfabrication processes, can be taken into account to calculate the macroscopic properties of the film.

The mathematical theory of homogenization (MTH) is a well established multiscale method that provides the equivalent homogeneous material properties for heterogeneous material when the separation of scales works [Bensoussan et al. 1978; Sanchez-Palencia and Zaoui 1987]. The MTH provides the deterministic model which the stochastic finite element scheme will be built upon.

Stochastic finite element methods can be classified into two main categories based on the kind of results they yield. Firstly, reliability methods aim at calculating the failure probability, and hence focus on the tails of the probability density function of the response. Secondly, other methods aim at calculating the probabilistic characterization of the response. In this category, some methods, such as that presented hereafter, determine only the first two statistical moments of the response. For details, see first two statistical moments of the response. For more details, the reader should refer to [Schueller 1997; 2001; Manohar and Ibrahim 1999; Sudret and der Kiureghian 2000; van den Nieuwenhof 2003].

Monte Carlo simulations have the major advantage that accurate statistical solutions can be obtained for any problem whose deterministic solution is known, since they statistically converge to the correct solution provided that a large number of simulations is employed. Indeed, the basic principle of direct Monte Carlo simulation is to generate a sampling of the input parameters according to their probability distributions and correlations. For each input sample, a deterministic finite element analysis is carried out, giving an output sample. Finally, a response sampling is obtained, from which the mean and the standard deviation of the response can be derived. The disadvantage of the direct Monte Carlo method is that it is usually extremely computationally demanding due to the very large number of analyses that have to take place. The convergence rate of the estimator does not only increase by increasing the number of samples but also by decreasing the variance $\sigma_{y}^{2}$. Variance reduction techniques exploit additional a priori information to reduce the necessary sample size $n$ for a specified confidence level. Stratification techniques widely used in practice, such as Latin hypercube sampling (LHS) [McKay et al. 1979], use conditional expectations to reduce the variance of the estimator.

The basics of the perturbation stochastic finite element method (PSFEM) are expounded in [Kleiber and Hien 1992]. This method consists in a deterministic analysis complemented by a sensitivity analysis with respect to the random parameters. This permits the development of a Taylor series expansion of the response, from which the mean and variance of the response can be derived knowing the mean, variance, and correlation structure of the random parameters. Depending on the expansion order of the Taylor series expansion (1 or 2), the statistical moments of the response are first or second-order accurate and the method is called the first-order second moment (FOSM) or second-order second moment (SOSM) method, respectively. The main advantages of the PSFEM are its simplicity and applicability to a wide range of problems at low cost. It has been used in static and dynamic elastic analyses [Hien and Kleiber 1990; Kleiber and Hien 1992], buckling analyses [Altus and Totry 2003], composite ply failure problems [Onkar et al. 2007], inelastic deformation studies [Doltsinis and Kang 2006], linear transient heat transfer problems [Hien and Kleiber 1997], the analysis of free vibration of composite cantilevers [Oh and Librescu 1997], nonlinear dynamics [Lei and Qiu 2000], and the study of eigenvalues of structures with uncertain boundary conditions [Huang et al. 2007]. Due to the Taylor series expansion, accurate results are expected only in case of relatively small variability of the parameters and for nearly linear problems. The derivatives of the structural matrices have to be calculated with respect to the random 
variables. This can be done analytically, semianalytically, or by finite difference. These computations can be time-consuming, particularly when the second-order terms are included.

The PSFEM has been applied to the homogenization of elastic properties of composite materials such as a bicomponent 1D bar [Kaminski 2000] and unidirectional fiber-reinforced composites [Kaminski and Kleiber 2000; Sakata et al. 2008]. All these applications use random variables which are related to inherent material properties of the composite components. This paper considers polycrystalline material and in particular accounts for the uncertainty resulting from the scattering of constitutive grain orientation. Moreover, the effect of the unit cell size on the macroscopic (homogenized) property variation is investigated as well as the geometry of the grains generated by Voronoi tessellation. The aim of this study is not only to quantify the uncertainty in the macroscopic polycrystalline elastic properties but also to gain confidence in the minimum size required to consider the unit cell as a representative volume element (RVE) of the polycrystalline material. An unit cell is considered as a RVE when the standard deviation of the homogenized properties is below a threshold defined by the analyst. In that case, the unit cell is stochastically representative of the material behavior, that is, the standard deviation of the output represents the uncertainty resulting from the input uncertainty.

After a review of the basics of the finite element implementation of the classical MTH in Section 2, the perturbation stochastic finite element-based homogenization formulation is derived by applying the PSFEM to the homogenization scheme in Section 3. In Section 4, the obtained methodology is first verified against Monte Carlo results for a unit cell of 4 grains in which one random variable is considered. Then, the methodology is applied on multigrain unit cells made of textured gold. This application investigates the influence of unit cell size, grain geometry, and orientation randomness on the macroscopic (homogenized) elastic properties.

\section{Multiscale model}

As described in the previous section, the focus of this work is on quantifying the stochastic nature of the elastic properties of polycrystalline media. More precisely, we aim at assessing how the uncertainties in the material texture (that is, the grain orientation and stiffness) impact its homogenized macroscopic elastic properties. As indicated earlier, the homogenization scheme adopted in this study is based on the finite element implementation of the classical mathematical theory of homogenization (MTH). In this section, the MTH is summarized and the grain-level anisotropic response is described.

Following the classic work [Bensoussan et al. 1978] as well as [Guedes and Kikuchi 1990], we start from a $y$-periodic asymptotic expansion of the displacement field

$$
\boldsymbol{u}(\boldsymbol{x}, \boldsymbol{y}) \approx \boldsymbol{u}^{(0)}(\boldsymbol{x}, \boldsymbol{y})+\xi^{1} \boldsymbol{u}^{(1)}(\boldsymbol{x}, \boldsymbol{y})+\xi^{2} \boldsymbol{u}^{(2)}(\boldsymbol{x}, \boldsymbol{y})+\ldots,
$$

where the superscripts in parentheses represent the level in the multiscale expansion, and $\boldsymbol{x}$ and $\boldsymbol{y}$ label the material points at the macro and microscale, respectively (see Figure 1). The relation between the two scales is defined by the asymptotic parameter $\xi$ as $\boldsymbol{y}=\boldsymbol{x} / \xi$, where $\xi$ roughly corresponds to the size of the periodic unit cell that serves as the basis of the homogenization scheme. Applying the chain rule,

$$
\frac{d \varphi(\boldsymbol{x}, \boldsymbol{y})}{d \boldsymbol{x}}=\frac{\partial \varphi(\boldsymbol{x}, \boldsymbol{y})}{\partial \boldsymbol{x}}+\frac{1}{\xi} \frac{\partial \varphi(\boldsymbol{x}, \boldsymbol{y})}{\partial \boldsymbol{y}},
$$




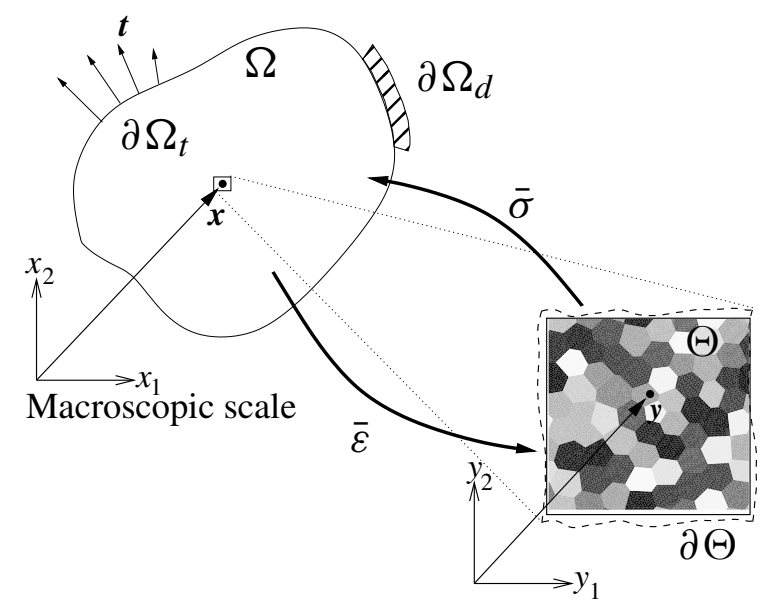

Microscopic scale

Figure 1. Schematic view of the homogenization scheme, with the microscopic scale showing the polycrystalline periodic unit cell.

we obtain the corresponding expansion for the strain tensor

$$
\varepsilon_{i j} \approx \xi^{-1}\left(\frac{\partial^{S} u_{i}^{(0)}}{\partial y_{j}}\right)+\xi^{0}\left(\frac{\partial^{S} u_{i}^{(0)}}{\partial x_{j}}+\frac{\partial^{S} u_{i}^{(1)}}{\partial y_{j}}\right)+\xi^{1}\left(\frac{\partial^{S} u_{i}^{(1)}}{\partial x_{j}}+\frac{\partial^{S} u_{i}^{(2)}}{\partial y_{j}}\right)+\ldots,
$$

where we adopt the symmetric gradient tensor notation

$$
\frac{\partial^{S} \bullet_{i}}{\partial x_{j}}=\frac{1}{2}\left(\frac{\partial \bullet_{i}}{\partial x_{j}}+\frac{\partial \bullet_{j}}{\partial x_{i}}\right)
$$

For the elastic problem characterized by a local fourth-order material stiffness tensor $D_{i j k l}$ and tractions $t$ applied along the portion $\partial \Omega_{t}$ of the boundary $\partial \Omega$, the principle of virtual work takes the form

$$
\int_{\Omega} D_{i j k l} \varepsilon_{k l} \frac{\partial^{S} v_{i}}{\partial x_{j}} d \Omega-\int_{\partial \Omega_{t}} t_{i} v_{i} d \Omega=0
$$

for all admissible displacements $v$ satisfying

$$
\boldsymbol{v} \in\left[H^{1}\right]^{2}, \quad \boldsymbol{v}=0 \text { on } \partial \Omega_{d}=\partial \Omega \backslash \partial \Omega_{t},
$$

where $\left[H^{1}\right]^{2}$ is the Sobolev space for the 2D problem. Combining (2-3) and (2-5) and grouping the terms by powers of $\xi$ leads to

$$
\begin{gathered}
\frac{1}{\xi^{2}} \int_{\Omega} D_{i j k l} \frac{\partial^{S} u_{k}^{(0)}}{\partial y_{l}} \frac{\partial^{S} v_{i}}{\partial y_{j}} d \Omega=0 \\
\frac{1}{\xi} \int_{\Omega} D_{i j k l}\left[\left(\frac{\partial^{S} u_{k}^{(0)}}{\partial x_{l}}+\frac{\partial^{S} u_{k}^{(1)}}{\partial y_{l}}\right) \frac{\partial^{S} v_{i}}{\partial y_{j}}+\frac{\partial^{S} u_{k}^{(0)}}{\partial y_{l}} \frac{\partial^{S} v_{i}}{\partial x_{j}}\right] d \Omega=0 \\
\int_{\Omega} D_{i j k l}\left[\left(\frac{\partial^{S} u_{k}^{(1)}}{\partial x_{l}}+\frac{\partial^{S} u_{k}^{(2)}}{\partial y_{l}}\right) \frac{\partial^{S} v_{i}}{\partial y_{j}}+\left(\frac{\partial^{S} u_{k}^{(0)}}{\partial x_{l}}+\frac{\partial^{S} u_{k}^{(1)}}{\partial y_{l}}\right) \frac{\partial^{S} v_{i}}{\partial x_{j}}\right] d \Omega=\int_{\partial \Omega_{t}} t_{i} v_{i} d \Omega .
\end{gathered}
$$


As shown in [Guedes and Kikuchi 1990], we establish from (2-7) that $\boldsymbol{u}^{(0)}$ is exclusively a function of $\boldsymbol{x}$, that is,

$$
\boldsymbol{u}^{(0)}(\boldsymbol{x}, \boldsymbol{y})=\boldsymbol{u}^{(0)}(\boldsymbol{x})
$$

while (2-8) leads to the following expression of the equilibrium at the microscale:

$$
\frac{1}{|\Theta|} \int_{\Theta} D_{i j k l}\left[\left(\frac{\partial^{S} u_{k}^{(0)}}{\partial x_{l}}+\frac{\partial^{S} u_{k}^{(1)}}{\partial y_{l}}\right) \frac{\partial^{S} v_{i}}{\partial y_{j}}\right] d \Theta=0
$$

where $\Theta$ denotes the volume of the periodic unit cell.

Defining the macroscopic strain $\bar{\varepsilon}=\partial^{S} \boldsymbol{u}^{(0)} / \partial \boldsymbol{x}$ and the fluctuation strain $\tilde{\varepsilon}=\partial^{S} \boldsymbol{u}^{(1)} / \partial \boldsymbol{y}$, we recast $(2-11)$ as

$$
\frac{1}{|\Theta|} \int_{\Theta} D_{i j k l} \tilde{\varepsilon}_{k l} \frac{\partial^{S} v_{i}}{\partial y_{j}} d \Theta=-\frac{1}{|\Theta|} \int_{\Theta} D_{i j k l} \frac{\partial^{S} v_{i}}{\partial y_{j}} d \Theta \bar{\varepsilon}_{k l},
$$

where we observe that the macroscopic strain serves as a loading term in the solution for the fluctuation strain. Relation (2-12) allows us to solve for the fluctuation strain at every point in the periodic unit cell, defining the strain localization operator $G_{i j k l}$ as

$$
\tilde{\varepsilon}_{i j}(\boldsymbol{x}, \boldsymbol{y})=G_{i j k l}(\boldsymbol{x}, \boldsymbol{y}) \bar{\varepsilon}_{k l} \text {. }
$$

Finally, the homogenized elastic properties of the polycrystalline material can be extracted from the equilibrium equation (2-9). Assuming that $v_{i}$ is a function of $\boldsymbol{x}$ only, (2-9) reduces to [Guedes and Kikuchi 1990]

$$
\int_{\Omega}\left[\frac{1}{|\Theta|} \int_{\Theta} D_{i j k l}\left(\bar{\varepsilon}_{k l}+\tilde{\varepsilon}_{k l}\right) d \Theta\right] \frac{\partial^{S} v_{i}}{\partial x_{j}} d \Omega=\int_{\partial \Omega} t_{i} v_{i} d \partial \Omega,
$$

or, combining with (2-13),

$$
\int_{\Omega}\left[\frac{1}{|\Theta|} \int_{\Theta} D_{i j k l}\left(I_{k l m n}+G_{k l m n}\right) d \Theta\right] \bar{\varepsilon}_{m n} \frac{\partial^{S} v_{i}}{\partial x_{j}} d \Omega=\int_{\partial \Omega} t_{i} v_{i} d \partial \Omega,
$$

where $I_{k l m n}=\frac{1}{2}\left(\delta_{k m} \delta_{l n}+\delta_{k n} \delta_{l m}\right)$. From (2-15), we identify the homogenized elastic tensor $D_{i j k l}^{h}$ as

$$
D_{i j m n}^{h}=\frac{1}{|\Theta|} \int_{\Theta} D_{i j k l}\left(I_{k l m n}+G_{k l m n}\right) d \Theta .
$$

For the finite element implementation of this homogenization scheme, it is more convenient to recast (2-16) using the Voigt formulation (that is, in matrix notation) as

$$
\boldsymbol{D}^{h}=\frac{1}{|\Theta|} \sum_{e=1}^{n} \int_{\Theta^{e}}\left(\boldsymbol{D}^{e} \boldsymbol{I}+\boldsymbol{D}^{e} \boldsymbol{G}^{e}\right) d \Theta
$$

where the domain $\Theta$ has been subdivided into $n$ elements and the superscript $e$ denotes the local value in element $e$. 
In this notation, the stiffness tensor of a material with cubic symmetry, such as the face centered cubic material of interest in this work, is given by

$$
\boldsymbol{D}_{c}^{e}=\left[\begin{array}{cccccc}
C_{11} & C_{12} & C_{12} & 0 & 0 & 0 \\
C_{12} & C_{11} & C_{12} & 0 & 0 & 0 \\
C_{12} & C_{12} & C_{11} & 0 & 0 & 0 \\
0 & 0 & 0 & C_{44} & 0 & 0 \\
0 & 0 & 0 & 0 & C_{44} & 0 \\
0 & 0 & 0 & 0 & 0 & C_{44}
\end{array}\right],
$$

in a coordinate system attached to each crystal (or grain) as denoted by the subscript $c$. To transform the stiffness matrix into the sample coordinate system (denoted by the subscript $s$ ), we adopt the Bunge convention and introduce the triad $\left(\phi_{1}, \theta, \phi_{2}\right)$, defining the individual rotation matrices

$$
\boldsymbol{R}_{1}=\left[\begin{array}{ccc}
\cos \phi_{1} & \sin \phi_{1} & 0 \\
-\sin \phi_{1} & \cos \phi_{1} & 0 \\
0 & 0 & 1
\end{array}\right], \quad \boldsymbol{R}_{2}=\left[\begin{array}{ccc}
1 & 0 & 0 \\
0 & \cos \theta & \sin \theta \\
0 & -\sin \theta & \cos \theta
\end{array}\right], \quad \boldsymbol{R}_{3}=\left[\begin{array}{ccc}
\cos \phi_{2} & \sin \phi_{2} & 0 \\
-\sin \phi_{2} & \cos \phi_{2} & 0 \\
0 & 0 & 1
\end{array}\right] .
$$

The resulting rotation matrix from the crystal coordinate system to the sample one is given by

$$
\boldsymbol{Q}=\left(\boldsymbol{R}_{3} \boldsymbol{R}_{2} \boldsymbol{R}_{1}\right)^{T}
$$

The rotation of the forth-order tensor $D_{i j m n}$ denoted $\boldsymbol{D}$ in matrix notation is then expressed as

$$
\boldsymbol{D}_{s}^{e}=\boldsymbol{K} \boldsymbol{D}_{c}^{e} \boldsymbol{K}^{T},
$$

while the compliance matrix $\boldsymbol{S}_{c}^{e}=\left(\boldsymbol{D}_{c}^{e}\right)^{-1}$ is transformed as

$$
\boldsymbol{S}_{s}^{e}=\left(\boldsymbol{K}^{-1}\right)^{T} \boldsymbol{S}_{c}^{e} \boldsymbol{K}^{-1} .
$$

In (2-20) and (2-21), $\boldsymbol{K}$ is given by [Ting 1996]

$$
\boldsymbol{K}=\left[\begin{array}{cc}
\boldsymbol{K}_{1} & 2 \boldsymbol{K}_{2} \\
\boldsymbol{K}_{3} & \boldsymbol{K}_{4}
\end{array}\right],
$$

where

$$
\begin{gathered}
\boldsymbol{K}_{1}=\left[\begin{array}{lll}
Q_{11}^{2} & Q_{12}^{2} & Q_{13}^{2} \\
Q_{21}^{2} & Q_{22}^{2} & Q_{23}^{2} \\
Q_{31}^{2} & Q_{32}^{2} & Q_{33}^{2}
\end{array}\right], \boldsymbol{K}_{2}=\left[\begin{array}{lll}
Q_{12} Q_{13} & Q_{13} Q_{11} & Q_{11} Q_{12} \\
Q_{22} Q_{23} & Q_{23} Q_{21} & Q_{21} Q_{22} \\
Q_{32} Q_{33} & Q_{33} Q_{31} & Q_{31} Q_{32}
\end{array}\right], \boldsymbol{K}_{3}=\left[\begin{array}{lll}
Q_{21} Q_{31} & Q_{22} Q_{32} & Q_{23} Q_{33} \\
Q_{31} Q_{11} & Q_{32} Q_{12} & Q_{33} Q_{13} \\
Q_{11} Q_{21} & Q_{12} Q_{22} & Q_{13} Q_{23}
\end{array}\right], \\
\boldsymbol{K}_{4}=\left[\begin{array}{lll}
Q_{22} Q_{33}+Q_{23} Q_{32} & Q_{23} Q_{31}+Q_{21} Q_{33} & Q_{21} Q_{32}+Q_{22} Q_{31} \\
Q_{32} Q_{13}+Q_{33} Q_{12} & Q_{33} Q_{11}+Q_{31} Q_{13} & Q_{31} Q_{12}+Q_{32} Q_{11} \\
Q_{12} Q_{23}+Q_{13} Q_{22} & Q_{13} Q_{21}+Q_{11} Q_{23} & Q_{11} Q_{22}+Q_{12} Q_{21}
\end{array}\right],
\end{gathered}
$$

with $Q_{i j}$ denoting the components of $\boldsymbol{Q}$ defined in (2-19). To perform the analysis in a plane-stress framework, the compliance matrix is first expressed in the sample coordinate system using (2-21). The 


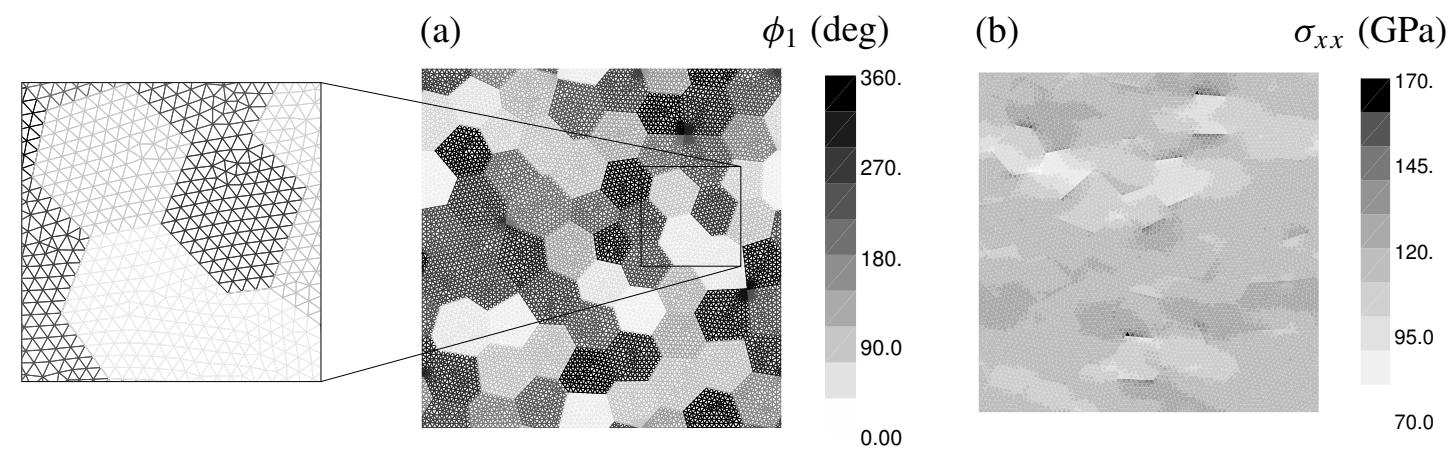

Figure 2. For a unit cell with 64 grains: (a) typical $\phi_{1}$ distribution and finite element mesh and (b) stress field $\left(\sigma_{x x}\right)$. The right figure emphasizes the heterogeneous nature of the stress field in the RVE.

plane-stress compliance matrix is then obtained as

$$
\boldsymbol{S}_{s, 2 \mathrm{D}}^{e}=\left[\begin{array}{lll}
S_{S, 11} & S_{S, 12} & S_{s, 16} \\
S_{S, 21} & S_{S, 22} & S_{s, 26} \\
S_{S, 61} & S_{S, 62} & S_{s, 66}
\end{array}\right],
$$

where $S_{s, a b}$ represents component $a b$ of the 3D compliance matrix $S_{s}^{e}$ given by (2-21). Finally, the stiffness matrix is found by inverting the compliance matrix:

$$
\boldsymbol{D}_{s, 2 \mathrm{D}}^{e}=\left(\boldsymbol{S}_{s, 2 \mathrm{D}}^{e}\right)^{-1} .
$$

As stated in Section 1, this work is motivated by the study of texturized gold thin films. In this particular case, the material constants are $C_{11}=186 \mathrm{GPa}, C_{12}=157 \mathrm{GPa}$, and $C_{44}=42 \mathrm{GPa}$. To represent the $\langle 111\rangle$ fiber texture only two Euler angles need to be specified, $\theta=54.7^{\circ}$ and $\phi_{2}=45^{\circ}$. The third angle $\phi_{1}$ is not defined and can assume any value, as all grains have the $\langle 111\rangle$ pole normal to the surface and the in-plane rotations are random. Here it is important to stress that the $\phi_{1}$ values used in the application cases in Section 4 have been randomly chosen. However, $\phi_{1}$ is not considered as a random variable of interest in this work since it does not describe the uncertainty of the material texture.

A typical result obtained with the multiscale scheme is presented in Figure 2 for the case of a 64-grain RVE and a macroscopic strain $\bar{\varepsilon}_{x x}$ equal to $1 \%$. The heterogeneous stress field due to angles mismatch for a macroscopic strain of $1 \%$ presents a ratio of approximately 2.4 between the maximum and minimum stress values in the RVE.

\section{Perturbation stochastic FE formulation}

A set of stochastic variables enter the finite element-based homogenization scheme described in previous section. These random variables, denoted hereafter by $\zeta$, include quantities such as the stiffness and orientation of the grains that compose the periodic unit cell. Our goal in this section is to derive the key quantities entering the PSFEM formulation of the MTH scheme summarized in Section 2. We start from 
the stochastic form of the homogenized stiffness matrix (2-17) which is given by

$$
\boldsymbol{D}^{h}(\zeta)=\frac{1}{|\Theta|} \sum_{e=1}^{n} \int_{\Theta^{e}}\left(\boldsymbol{D}^{e}(\zeta) \boldsymbol{I}+\boldsymbol{D}^{e}(\zeta) \boldsymbol{G}^{e}(\zeta)\right) d \Theta
$$

where $\boldsymbol{G}$ implicitly depends on the uncertainty through

$$
\frac{1}{|\Theta|} \int \boldsymbol{B}^{T} \boldsymbol{D}(\zeta) \boldsymbol{G}^{e}(\zeta) d \Theta=\frac{1}{|\Theta|} \int \boldsymbol{B}^{T} \boldsymbol{D}(\zeta) d \Theta,
$$

with $\boldsymbol{B}$ denoting the traditional strain-displacement matrix.

The perturbation method considers the random design variables $b_{i}$ as perturbed from their expectation $\bar{b}_{i}$, so that the random variables $b_{i}$ are written as the sum of a deterministic value $\bar{b}_{i}$ and a zero-mean random variable $\Delta b_{i}$ as

$$
b_{i}=\bar{b}_{i}+\Delta b_{i}
$$

The covariance matrix $\boldsymbol{C}$ of the random variables $b_{i}$ is related to the zero-mean random variables as

$$
C_{i j}=\operatorname{Cov}\left(b_{i}, b_{j}\right)=E\left[\Delta b_{i} \Delta b_{j}\right] .
$$

The perturbation method consists in expanding the random quantities about their expectations via a truncated Taylor series expansion. The second-order Taylor expansion about the nominal value $\overline{\boldsymbol{b}}$ with respect to the random variables $b_{i}$ is given by

$$
\boldsymbol{D}^{h}(\overline{\boldsymbol{b}}) \approx \overline{\boldsymbol{D}}^{h}+\sum_{i=1}^{n} \boldsymbol{D}_{, i}^{h} \Delta b_{i}+\frac{1}{2} \sum_{i=1}^{n} \sum_{j=1}^{n} \boldsymbol{D}_{, i j}^{h} \Delta b_{i} \Delta b_{j}
$$

where the subscript,$i$ denotes the derivative with respect to random variable $b_{i}$. Since the random variables $\Delta b_{i}$ are zero-mean random variables of known covariance, the expectation of the homogenized property matrix is

$$
E\left[\boldsymbol{D}^{h}(\overline{\boldsymbol{b}})\right] \approx \overline{\boldsymbol{D}}^{h}+\sum_{i=1}^{n} \boldsymbol{D}_{, i}^{h} E\left[\Delta b_{i}\right]+\frac{1}{2} \sum_{i=1}^{n} \sum_{j=1}^{n} \boldsymbol{D}_{, i j}^{h} E\left[\Delta b_{i} \Delta b_{j}\right]=\overline{\boldsymbol{D}}^{h}+\frac{1}{2} \sum_{i=1}^{n} \sum_{j=1}^{n} \boldsymbol{D}_{, i j}^{h} \operatorname{Cov}\left(b_{i}, b_{j}\right),
$$

while the variance of the homogenized property matrix is expressed as

$$
\operatorname{Var}\left(\boldsymbol{D}^{h}(\overline{\boldsymbol{b}})\right)=E\left[\left(\boldsymbol{D}^{h}(\overline{\boldsymbol{b}})-E\left[\boldsymbol{D}^{h}(\overline{\boldsymbol{b}})\right]\right)^{2}\right] \approx \sum_{i=1}^{n} \sum_{j=1}^{n} \boldsymbol{D}_{, i}^{h} \boldsymbol{D}_{, j}^{h} E\left[\Delta b_{i} \Delta b_{j}\right]=\sum_{i=1}^{n} \sum_{j=1}^{n} \boldsymbol{D}_{, i}^{h} \boldsymbol{D}_{, j}^{h} \operatorname{Cov}\left(b_{i}, b_{j}\right) .
$$

The mean is second-order accurate with no first-order term in its expression, while the variance is first-order accurate since no second-order term appears in its expression. The variance expression will be the same whether a first-order or a second-order approximation is used in the perturbation method.

The first-order sensitivity of the homogenized property matrix has to be computed. Differentiating (2-17) with respect to $b_{i}$ gives

$$
\boldsymbol{D}_{, i}^{h}(\zeta)=\sum_{e=1}^{n} \int_{\Theta_{e}}\left(\boldsymbol{D}_{, i}^{e}(\zeta) \boldsymbol{I}+\boldsymbol{D}_{, i}^{e}(\zeta) \boldsymbol{G}^{e}(\zeta)+\boldsymbol{D}^{e}(\zeta) \boldsymbol{G}_{, i}^{e}(\zeta)\right) d \Theta
$$


where $\boldsymbol{G}_{, i}$ is computed by differentiating (3-2) as

$$
\int \boldsymbol{B}^{T} \boldsymbol{D}(\zeta) \boldsymbol{G}_{, i}(\zeta) d \Theta=\int \boldsymbol{B}^{T} \boldsymbol{D}_{, i}(\zeta) d \Theta-\int \boldsymbol{B}^{T} \boldsymbol{D}_{, i}(\zeta) \boldsymbol{G}(\zeta) d \Theta .
$$

Similarly, (3-8) gives the second-order sensitivity of $\boldsymbol{D}^{h}$ :

$$
\begin{aligned}
\boldsymbol{D}_{, i j}^{h}(\zeta)=\sum_{e=1}^{n} \int_{\Theta^{e}} & \left(\boldsymbol{D}_{, i j}^{e}(\zeta) \boldsymbol{I}+\boldsymbol{D}_{, i j}^{e}(\zeta) \boldsymbol{G}^{e}(\zeta)\right) d \Theta \\
& +\sum_{e=1}^{n} \int_{\Theta^{e}}\left(\boldsymbol{D}_{, i}^{e}(\zeta) \boldsymbol{G}_{, j}^{e}(\zeta)+\boldsymbol{D}_{, j}^{e}(\zeta) \boldsymbol{G}_{, i}^{e}(\zeta)\right) d \Theta+\sum_{e=1}^{n} \int_{\Theta^{e}}\left(\boldsymbol{D}^{e}(\zeta) \boldsymbol{G}_{, i j}^{e}(\zeta)\right) d \Theta
\end{aligned}
$$

where $\boldsymbol{G}_{, i j}$ is computed by differentiating (3-9)

$$
\begin{aligned}
\int \boldsymbol{B}^{T} \boldsymbol{D}(\zeta) \boldsymbol{G}_{, i j}(\zeta) d \Theta=\int \boldsymbol{B}^{T} \boldsymbol{D}_{, i j}(\zeta) d \Theta & -\int \boldsymbol{B}^{T} \boldsymbol{D}_{, i j}(\zeta) \boldsymbol{G}(\zeta) d \Theta \\
& -\int \boldsymbol{B}^{T} \boldsymbol{D}_{, i}(\zeta) \boldsymbol{G}_{, j}(\zeta) d \Theta-\int \boldsymbol{B}^{T} \boldsymbol{D}_{, j}(\zeta) \boldsymbol{G}_{, i}(\zeta) d \Theta .
\end{aligned}
$$

The quantities of interest, that is, the expectation of the homogenized property (3-6) and its variance (3-7), are obtained by evaluating (3-8) and (3-10) in a finite element framework.

\section{Verification}

The second-order PSFEM is applied to analyze the influence of uncertainty on the behavior of a cubic material in $\langle 111\rangle$ texture. The objective of this section is to verify the PSFEM formulation by comparing its results against Monte Carlo (MC) simulations. For this purpose, we chose to study a small unit cell composed of only 4 grains, shown in Figure 3.

Using the material constants introduced at the end of Section 2, the 2D nominal stiffness matrix of each grain is given by

$$
\overline{\boldsymbol{D}}^{c}=\left[\begin{array}{ccc}
186 & 157 & 0 \\
157 & 186 & 0 \\
0 & 0 & 42
\end{array}\right] \quad(\mathrm{GPa})
$$

expressed in its material axes. The Euler angles are set to $\theta=54.7^{\circ}$ and $\phi_{2}=45^{\circ}$, and $\phi_{1}$ has randomly assigned values of $188^{\circ}, 289^{\circ}, 294^{\circ}$, and $68^{\circ}$ for grains $1,2,3$, and 4 , respectively. $C_{11}$, the first

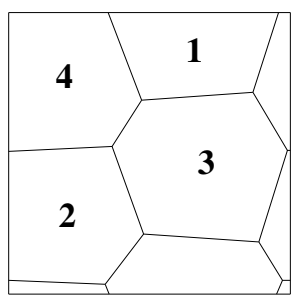

Figure 3. Periodic unit cell composed of 4 grains used to perform the verification test case of PSFEM. 


\begin{tabular}{|ccccc|}
\hline $\operatorname{CoV}(\%)$ & $\begin{array}{c}E\left[C_{11}^{h}\right](\mathrm{GPa}) \\
(\mathrm{PSFEM})\end{array}$ & $\begin{array}{c}E\left[C_{11}^{h}\right](\mathrm{GPa}) \\
(\mathrm{MC}, 2000 \text { samples })\end{array}$ & $\begin{array}{c}\sqrt{\operatorname{Var}\left[C_{11}^{h}\right]}(\mathrm{GPa}) \\
(\mathrm{PSFEM})\end{array}$ & $\begin{array}{c}\sqrt{\operatorname{Var}\left[C_{11}^{h}\right]}(\mathrm{GPa}) \\
(\mathrm{MC}, 2000 \text { samples })\end{array}$ \\
2 & 120.0338 & 120.0052 & 0.5410 & 0.5529 \\
4 & 120.0235 & 119.8789 & 1.0820 & 1.1930 \\
6 & 120.0133 & 119.6227 & 1.6229 & 2.1429 \\
\hline
\end{tabular}

Table 1. Verification test case based on Figure 3 unit cell: PSFEM and MC results for coefficients of variance of $2 \%, 4 \%$, and $6 \%$ of the random variable $C_{11}$ of grain 1 .

component of the stiffness matrix expressed in the material axes of grain 1, is considered as a Gaussian random variable. The effect of the level of uncertainty is studied by successively considering a coefficient of variation, $\operatorname{CoV}\left(C_{11}\right)$, of $2 \%, 4 \%$, and $6 \%$.

The unit cell is loaded with a tensile strain in direction 1 of the sample coordinate system. This loading allows us to quantify the effect of uncertainty on the first column of the homogenized stiffness matrix expressed in the unit cell axes. The mean and the standard deviation of the homogenized stiffness matrix of the unit cell are computed using (3-6) and (3-7). In this paper, the results are presented for the first component of the homogenized stiffness matrix, $C_{11}^{h}$.

In order to verify the validity of the PSFEM approach, the results are compared to the MC results. A Latin hypercube sampling (LHS) method is used to generate 2000 samples of $C_{11}$ whose mean and standard deviation correspond to the values specified in the PSFEM study. LHS generates samples of a normally distributed random variable $s$ with a mean of zero and a standard deviation of one. $C_{11}$ is then expressed as a function of $s$ :

$$
C_{11}=\bar{C}_{11}\left(1+\operatorname{CoV}\left(C_{11}\right) s\right) .
$$

Then, the homogenized stiffness matrix is computed for each value of $C_{11}$. Finally, the mean and standard deviation of the 2000 samples of $C_{11}^{h}$ are computed and compared to PSFEM results.

Table 1 compares the PSFEM and MC values of the mean and standard deviation of $C_{11}^{h}$ for the three values of the coefficient of variation. The nominal value of $C_{11}^{h}$ is equal to $120.044 \mathrm{GPa}$, that is, the value of the deterministic analysis. As the coefficient of variation increases, the mean value increasingly deviates from the nominal value even if this difference is not significant. This variation is a quadratic function, as explained previously and shown in Figure 4, left.

Figure 4, right, shows the variation of the relative error of the mean and standard deviation of $C_{11}^{h}$ computed by PSFEM with respect to MC values, which are considered as reference values. PSFEM estimation error in both the mean and the standard deviation increases as the coefficient of variation increases because the approximation of the variation of $C_{11}^{h}$ as a quadratic function with respect to the uncertainty becomes less appropriate as the level of uncertainty increases.

The error in the mean is smaller than in the standard deviation. This is explained by the fact that the mean estimation is second-order accurate while the standard deviation estimation is only first-order accurate. The error is due to the fact that PSFEM approximates the variation of $C_{11}^{h}$ as a second-order polynomial function while, as shown in the left part of Figure 4, the actual variation of $C_{11}^{h}$ is more complex. This approximation is thus adequate in the vicinity of the nominal value. 

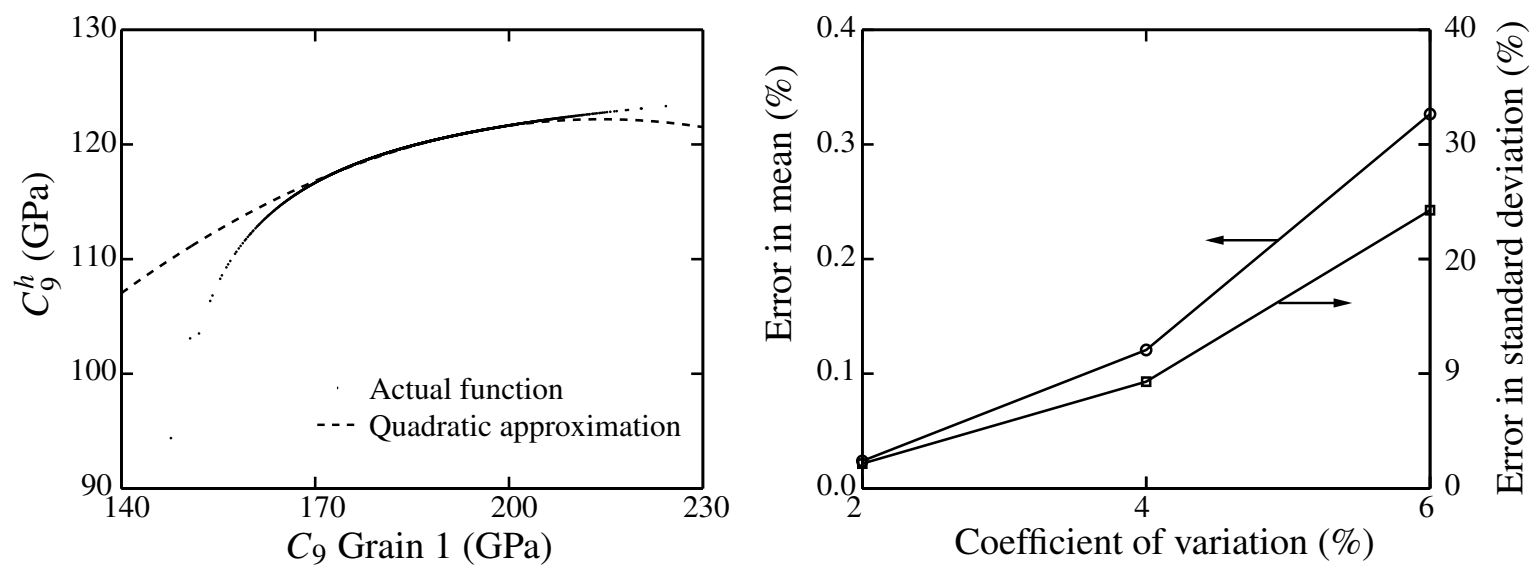

Figure 4. Verification test case based on Figure 3 unit cell. Left: variation of $C_{11}^{h}$ as a function of the random variable, that is, $C_{11}$ of grain 1. Right: percentage errors of $C_{11}^{h}$ mean and standard deviation between MC and PSFEM analyses for various $C_{11}$ coefficients of variation.

Another verification test case is to analyze the unit cell represented in Figure 3 assuming $C_{11}$ of each grain to be a random variable with a coefficient of variation of $3 \%$. The uncertainty is thus characterized by 4 random variables. The correlation between them is modeled by a correlation matrix. Three different correlation matrices are successively investigated. First, the correlation is supposed to be a matrix full of ones, which means that the four random variables are fully correlated and represent one and only one random variable. The opposite case is to set the correlation matrix to be the identity matrix, which is equivalent to considering 4 independent uncorrelated random variables. An intermediate correlation matrix is also studied:

$$
\left[\begin{array}{cccc}
1 & 0.5 & 0.25 & 0 \\
0.5 & 1 & 0.5 & 0.25 \\
0.25 & 0.5 & 1 & 0.5 \\
0 & 0.25 & 0.5 & 1
\end{array}\right]
$$

Note that such a correlation matrix could result from the representation of $C_{11}$ as a random field.

Both the mean and standard deviation increase as the correlation of the random variables increases, as can be observed in Figure 5. The reduction of the variance due to the fact that the random parameter is modeled by a random field instead of one random variable is also called the compensation effect on the variability. The verification demonstrates that PSFEM is a suitable tool to quantify the uncertainty of homogenized properties due to microstructural uncertainty. This method also allows us to evaluate the effect of different levels of correlation between the random variables on the macroscopic response.

\section{Study of geometry and material uncertainty}

The second-order PSFEM is applied to the analysis of several unit cells of a cubic material with a $\langle 111\rangle$ texture. As explained in Section 2, for $\langle 111\rangle$ texture, the Euler angles are $\theta=54.7^{\circ}$ and $\phi_{2}=45^{\circ}$, and $\phi_{1}$ has random assigned values. The influence of uncertainty in the $\theta$ angle of each grain is studied on 


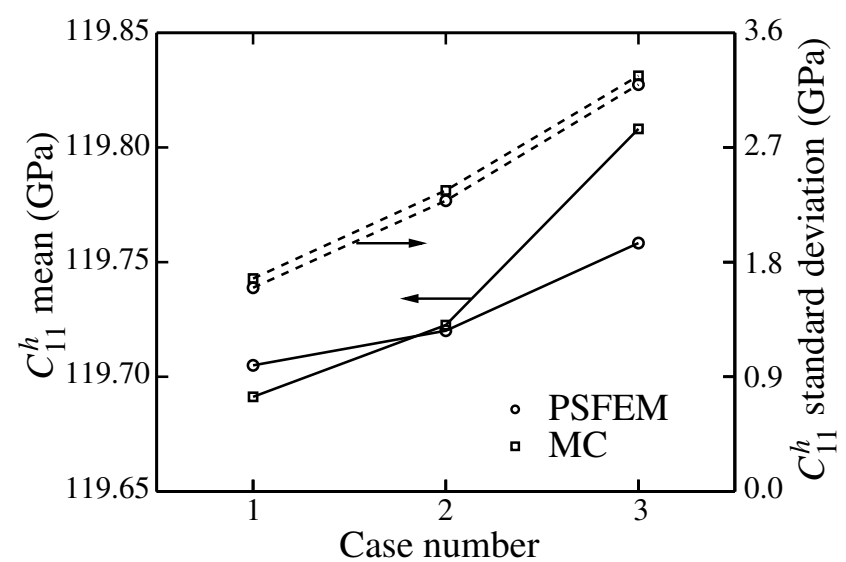

Figure 5. Effect of the correlation of random variables on the mean and standard deviation of $C_{11}^{h}$. Case 1: four independent random variables; case 2: correlation matrix (4-3); case 3: one random variable.

the material behavior of periodic unit cells from 4 to 64 grains. In particular, the mean and standard deviation of the homogenized $C_{11}^{h}$ are quantified.

5A. Influence of material texture uncertainty. To study the influence of texture uncertainty, a unit cell with 16 grains and 16 random variables (one per grain) is generated. The angle $\theta$ which controls the perpendicularity of the $\langle 111\rangle$ pole with respect to the surface of the thin film is chosen as the random variable. The random variables are assumed to be independent. The standard deviation of $\theta$ is varied from $2^{\circ}$ to $10^{\circ}$. In each case, the mean and standard deviation of $C_{11}^{h}$ of the unit cell are computed using PSFEM and are compared to the values obtained from MC analysis using 15000 samples. The same finite element model, that is, with the same mesh and grain geometry, is used for the PSFEM and MC analyses.

Figure 6 shows that as the standard deviation increases, the error of the PSFEM approximation increases. The error on the mean remains lower than one percent while the error on the standard deviation varies from $0.73 \%$ to $9.84 \%$. As explained previously, PSFEM method relies on a second-order approximation of the response, and so its accuracy is limited within a neighborhood of the expectation value of the random variable. This analysis shows that for the particular problem of interest, the error on the PSFEM is less than $0.73 \%$ for the mean and standard deviation when $\theta$ has a standard deviation of at most $2^{\circ}$. The error on the means remains low for $\theta$ standard deviation up to $10^{\circ}$, that is, less than one percent, while the error on the standard deviation becomes not negligible, that is, above $5 \%$, once the $\theta$ standard deviation reaches $7^{\circ}$. This analysis allows us to quantify the limitation of PSFEM on the quantification of the influence of material texture uncertainty.

It should be noted that the CPU requirement associated with the PSFEM is substantially lower than for the MC method since considerably less homogenization problems have to be solved. Therefore, even if PSFEM approximates the response statistics, it allows us to gain valuable knowledge on the macroscopic property uncertainty induced by grain-level uncertainties at a lower cost than the classical MC method. However, it should be noted that if the number of random variables becomes really large, PSFEM's computational cost may be such that using the MC method would be more advantageous. 


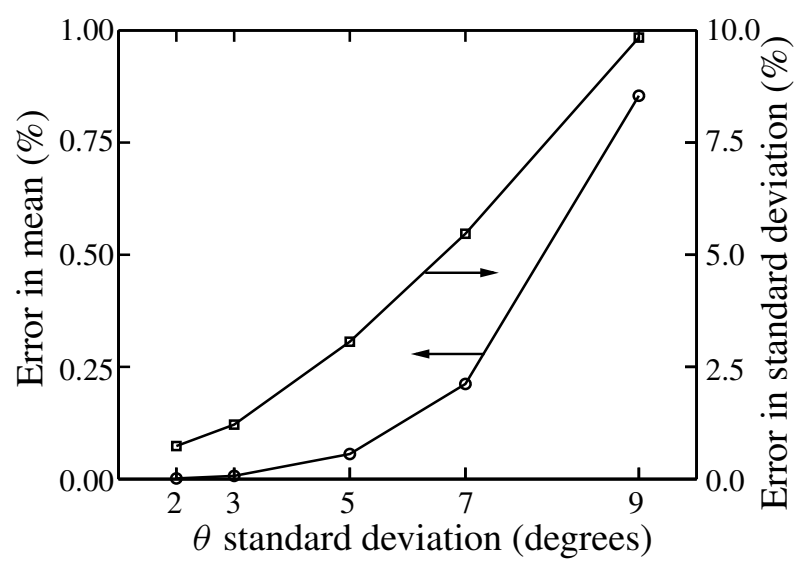

Figure 6. Material texture uncertainty study: Percentage errors of $C_{11}^{h}$ mean and standard deviation between MC and PSFEM analyses for various $\theta$ standard deviations on a 16 grain unit cell.

To study the effect of the unit cell size, for a fixed standard deviation of $\theta$, the number of grains of the unit cell is varied from 4 to 64. For each unit cell, the mean and standard deviation of $C_{11}^{h}$ are evaluated using PSFEM and the MC method. For each unit cell size, MC analyses have been performed for three LHS sets of 8000, 12000, and 15000 samples, respectively. The statistics for $C_{11}^{h}$ were obtained from those of the three sets. The results of both methodologies are presented in Figure 7. The maximum error on the mean is $0.0084 \%$ while for the standard deviation it is $8.1 \%$. As the unit cell size increases, the standard deviation of $C_{11}^{h}$ decreases up to a threshold of $0.31 \mathrm{GPa}$. The influence of the unit cell size on the standard deviation of $C_{11}^{h}$ provides an important parameter to decide to what extent a unit cell can be considered as a RVE. Once the standard deviation of the output reaches a threshold, the unit cell

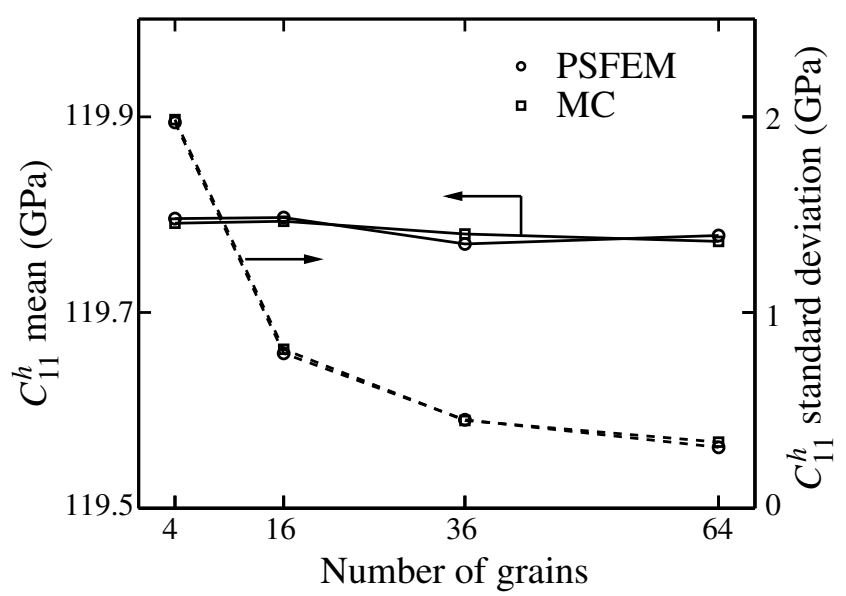

Figure 7. Material texture uncertainty study: Effect of unit cell size on MC and PSFEM prediction of the mean (solid curves) and standard deviation (dashed curves) for $C_{11}^{h}$ with a standard deviation of $2^{\circ}$ in $\theta$. 


\begin{tabular}{|ccc|}
\hline Parameter & Mean $(\mathrm{GPa})$ & Standard deviation $(\mathrm{GPa})$ \\
$C_{11}^{h}$ & 119.782 & 0.311 \\
$C_{12}^{h}$ & 68.2993 & 0.088 \\
$C_{21}^{h}$ & 68.2993 & 0.088 \\
$C_{22}^{h}$ & 119.7785 & 0.3356 \\
\hline
\end{tabular}

Table 2. PSFEM mean and standard deviation of effective components of homogenized stiffness matrix of a unit cell composed of 64 grains with a standard deviation of $2^{\circ}$ in $\theta$.

can be considered stochastically representative, that is, the standard deviation threshold value represents the uncertainty resulting from the input uncertainty. For this particular case, when the unit cell contains more than 64 grains, it is observed that a textural uncertainty, characterized by a $2^{\circ}$ standard deviation, introduces an uncertainty on $C_{11}^{h}$ characterized by a $0.31 \mathrm{GPa}$ standard deviation.

All aforepresented results relate to $C_{11}^{h}$. Table 2 lists the mean and standard deviation obtained for other effective components of the homogenized stiffness matrix. The results are for a unit cell composed of 64 grains. For each grain, its $\theta$ angle is considered as a random variable characterized by a $2^{\circ}$ standard deviation. These 64 random variables are considered as independent, which means that their correlation matrix is equal to the identity matrix. The uncertainty introduced on $C_{11}^{h}$ and $C_{22}^{h}$ are similar: $0.31 \mathrm{GPa}$ and $0.33 \mathrm{GPa}$, respectively. Conclusions based on one or on the other would be the same. The means of $C_{12}^{h}$ and $C_{21}^{h}$ are equal as well as their standard deviation. The relative importance of the standard deviation with respect to the mean is twice larger on the diagonal components than on the off-diagonal ones. Based on these facts, the study will hereafter focus on $C_{11}^{h}$ results.

5B. Combining PSFEM and Monte Carlo methods to evaluate geometry and texture uncertainty. So far, only uncertainty in the material parameters has been considered and PSFEM and MC analyses have been carried out on only one representation of the unit cell. In order to take into account the geometric uncertainty of grains as well as the grains material property randomness, MC and PSFEM are coupled. A standard deviation of $10^{\circ}$ is considered for $\theta$. The geometry variation is taken into account by using the Monte Carlo method. For each geometry sample, a PSFEM analysis is carried out in order to take into account the material property uncertainty. Using the law of total expectation, the output mean is computed by averaging over the geometry samples the mean obtained by PSFEM:

$$
\bar{S}=\frac{\sum_{i_{\text {geo }}}^{n_{\text {geo }}} \bar{S}_{\text {PSFEM }}}{n_{\text {geo }}} .
$$

By applying the law of total variance, we get the value of the variance of the output as

$$
\operatorname{Var}(S)=\operatorname{Var}\left(\bar{S}_{\text {PSFEM }}\right)+E\left[\operatorname{Var}(S)_{\text {PSFEM }}\right] .
$$

It is assumed that the randomness of the material properties and the grain geometry are independent.

Monte Carlo analyses are performed for unit cells composed of 4, 16, 36, and 64 grains. For each unit cell size, 100 different geometry samples are generated by an in-house Voronoi tessellation software which can control the mean size of the grains. In this study, the mean grain size is defined as $21.36 \mathrm{~nm}$. The grain size is defined as the diameter of a circle whose area is equal to the grain's area. The randomness 


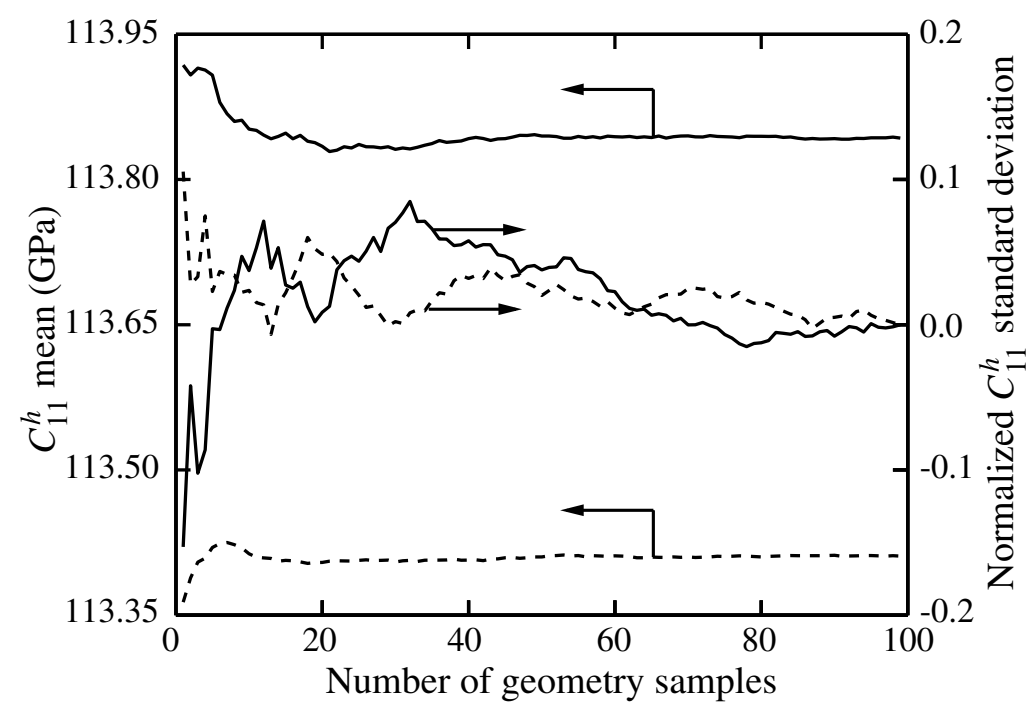

Figure 8. Geometry and texture uncertainty study: Variation of $C_{11}^{h}$ mean and normalized standard deviation for unit cell composed of 4 (solid curves) and 64 (dotted curves) grains with a standard deviation of $10^{\circ}$ in $\theta$.

on the grain distribution inside the unit cell is obtained by randomly assigning the seed point of the Voronoi tessellation.

Figure 8 presents the mean and standard deviation of $C_{11}^{h}$ versus the number of geometry samples considered for the unit cells of 4 and 64 grains. The standard deviation was normalized with respect to the standard deviations obtained using 100 samples. Those standard deviations were $9.86 \mathrm{GPa}$ and $1.56 \mathrm{GPa}$ for 4 and 64 grains, respectively. 20 geometric samples are sufficient to get a converged mean. For the standard deviation, convergence occurs from 80 samples. In the following, results are those obtained from 100 samples since they can be assumed as converged.

Figure 9 presents the mean and standard deviation of $C_{11}^{h}$ when considering geometric and material uncertainties. It is observed that the mean oscillates between 113 and $114 \mathrm{GPa}$, while the standard deviation decreases as the number of grains in the unit cell increases. The standard deviation is an important parameter for deciding to what extent a unit cell can be considered as a RVE. From this graph, it is concluded that for unit cells composed of 64 or more grains, the uncertainty in the homogenized properties is less than $1.56 \mathrm{GPa}$, that is, about $1.4 \%$ of the $C_{11}^{h}$ mean. From a practical point of view, this application shows that a unit cell of 64 grains can be considered as an RVE for the quantification of the uncertainty of the macroscopic elastic properties of a polycrystalline material induced by uncertainties on its grain geometry and texture.

To estimate the uncertainty of the geometry, the approximated grain size probability density function is plotted in Figure 10. The probability density is obtained by discretizing the grain size into 20 segments. The number of grains in each segment was counted and a value of the area of that segment was obtained; these values were normalized by the total area of the histogram. Figure 10 shows that the probability density functions for unit cells of 16 or more grains are similar. This guarantees that the comparison between the unit cells with 16, 36, and 64 grains is meaningful since all of them have the same input 


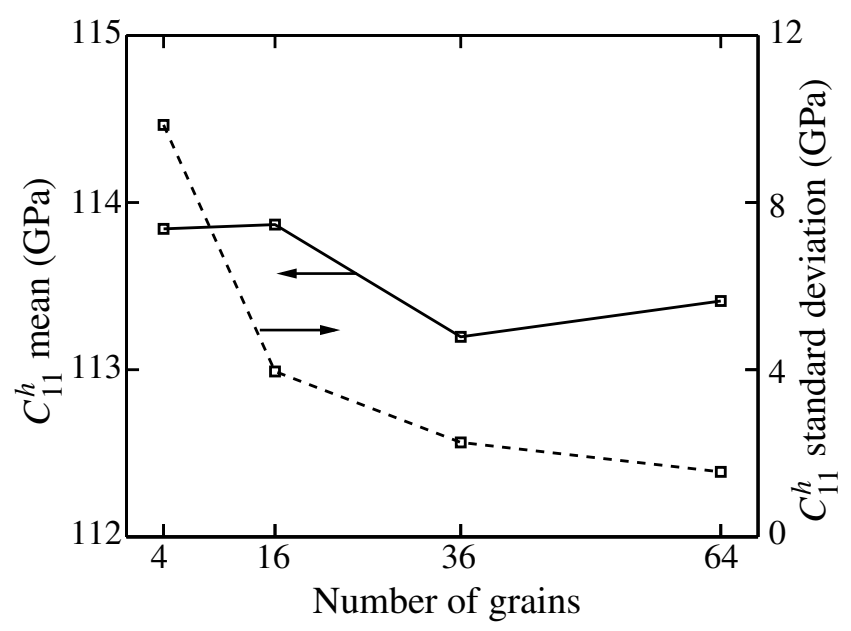

Figure 9. Geometry and texture uncertainty study: Effect of the unit cell size on $C_{11}^{h}$ mean (solid curves) and standard deviation (dashed curves) with a standard deviation of $10^{\circ}$ in $\theta$.

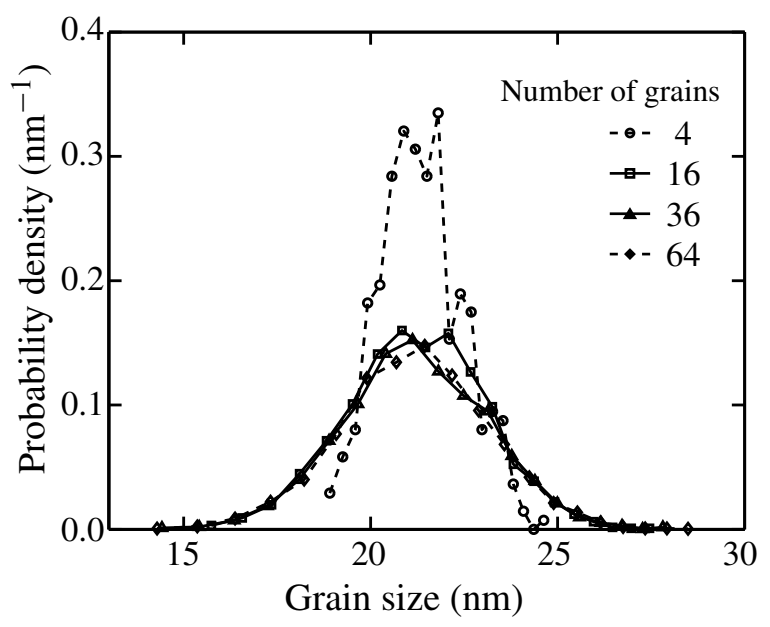

Figure 10. Probability density of grain size distribution for various RVE units. The mean grain sizes are 21.41,21.36, 21.36, and $21.35 \mathrm{~nm}$ and the standard deviations are $8.03,16.09,18.04$, and $19.30 \mathrm{~nm}$ for the unit cells composed of $4,16,36$, and 64 grains, respectively.

uncertainty. So the differences in mean and standard deviation in this case can be attributed to the set of grain orientations and the unit cell size.

\section{Conclusion}

This work presents the application of a perturbation-based stochastic method in the study of uncertainty in homogenized elastic properties of polycrystalline materials. The perturbation stochastic finite element 
method (PSFEM) has been applied to the mathematical theory of homogenization. The formulation obtained has been used to study the influence of geometry and material texture uncertainties at the grain level on the macroscopic polycrystalline material properties.

By comparing the results with Monte Carlo results on verification test cases, it has been shown that the PSFEM approximation errors depend on the problem of interest and the coefficient of variation for the input random variables. In the case of homogenization of gold polycrystalline structures, the limitation of PSFEM on the computation of the influence of material texture uncertainty has been quantified. The error on the $C_{11}^{h}$ standard deviation remains below $5 \%$ for a standard deviation in the Euler angle defining the $\langle 111\rangle$ texture up to $7^{\circ}$.

By combining Monte Carlo and PSFEM, the uncertainty of the geometry of the grains has been taken into account on top of the material texture uncertainty. The convergence of this approach has been demonstrated by numerical examples. The results show that for gold thin films, a unit cell with 64 grains or more predicts an uncertainty characterized by a coefficient of variation of $1.4 \%$ on $C_{11}^{h}$ for a standard deviation of $10^{\circ}$ in the Euler angle defining the $\langle 111\rangle$ texture.

The proposed approach is a general tool for quantifying the uncertainty of the elastic properties of polycrystalline materials as a function of the grain geometric and material properties and the unit cell size. Moreover, this study can provide better confidence on the necessary representative volume element size.

\section{Acknowledgments}

The authors gratefully acknowledge the support from DARPA through the MEMS/NEMS S\&T Fundamentals Program, from the Beckman Institute of Advanced Science and Technology, from NSF through Grant CMMI 09-27149 ARRA and from the Illinois Space Grant Consortium.

\section{References}

[Altus and Totry 2003] E. Altus and E. M. Totry, "Buckling of stochastically heterogeneous beams, using a functional perturbation method", Int. J. Solids Struct. 40:23 (2003), 6547-6565.

[Bensoussan et al. 1978] A. Bensoussan, J.-L. Lions, and G. Papanicolaou, Asymptotic analysis for periodic structures, Studies in Mathematics and its Applications 5, North-Holland, Amsterdam, 1978.

[Doltsinis and Kang 2006] I. Doltsinis and Z. Kang, "Perturbation-based stochastic FE analysis and robust design of inelastic deformation processes", Comput. Methods Appl. Mech. Eng. 195:19-22 (2006), 2231-2251.

[Guedes and Kikuchi 1990] J. M. Guedes and N. Kikuchi, "Preprocessing and postprocessing for materials based on the homogenization method with adaptive finite element methods", Comput. Methods Appl. Mech. Eng. 83:2 (1990), 143-198.

[Hien and Kleiber 1990] T. D. Hien and M. Kleiber, "Finite element analysis based on stochastic Hamilton variational principle", Comput. Struct. 37:6 (1990), 893-902.

[Hien and Kleiber 1997] T. D. Hien and M. Kleiber, "Stochastic finite element modelling in linear transient heat transfer", Comput. Methods Appl. Mech. Eng. 144:1-2 (1997), 111-124.

[Huang et al. 2007] B. Huang, Q. S. Li, W. H. Shi, and Z. Wu, "Eigenvalues of structures with uncertain elastic boundary restraints", Appl. Acoust. 68:3 (2007), 350-363.

[Kaminski 2000] M. Kaminski, "Homogenization of 1D elastostatics by the stochastic second order approach", Mech. Res. Commun. 27:3 (2000), 273-280.

[Kaminski and Kleiber 2000] M. Kaminski and M. Kleiber, "Perturbation based stochastic finite element method for homogenization of two-phase elastic composites”, Comput. Struct. 78:6 (2000), 811-826. 
[Kleiber and Hien 1992] M. Kleiber and T. D. Hien, The stochastic finite element method: basic perturbation technique and computer implementation, Wiley, Chichester, 1992.

[Lei and Qiu 2000] Z. Lei and C. Qiu, "A stochastic variational formulation for nonlinear dynamic analysis of structure", Comput. Methods Appl. Mech. Eng. 190:5-7 (2000), 597-608.

[Manohar and Ibrahim 1999] C. S. Manohar and R. A. Ibrahim, "Progress in structural dynamics with stochastic parameter variations: 1987-1998", Appl. Mech. Rev. (ASME) 52:5 (1999), 177-197.

[McKay et al. 1979] M. D. McKay, R. J. Beckman, and W. J. Conover, "A comparison of three methods for selecting values of input variables in the analysis of output from a computer code", Technometr. 21:2 (1979), 239-245.

[van den Nieuwenhof 2003] B. van den Nieuwenhof, Stochastic finite elements for elastodynamics: random field and shape uncertainty modelling using direct and modal perturbation-based approaches, Ph.D. thesis, Catholic University of Louvain, 2003, available at http://hdl.handle.net/2078.1/4995.

[Oh and Librescu 1997] D. H. Oh and L. Librescu, "Free vibration and reliability of composite cantilevers featuring uncertain properties", Reliab. Eng. Syst. Saf. 56:3 (1997), 265-272.

[Onkar et al. 2007] A. K. Onkar, C. S. Upadhyay, and D. Yadav, "Probabilistic failure of laminated composite plates using the stochastic finite element method", Compos. Struct. 77:1 (2007), 79-91.

[Sakata et al. 2008] S. Sakata, F. Ashida, T. Kojima, and M. Zako, "Three-dimensional stochastic analysis using a perturbationbased homogenization method for elastic properties of composite material considering microscopic uncertainty", Int. J. Solids Struct. 45:3-4 (2008), 894-907.

[Sanchez-Palencia and Zaoui 1987] E. Sanchez-Palencia and A. Zaoui (editors), Homogenization techniques for composite media (Udine, 1985), Lecture Notes in Physics 272, Springer, Berlin, 1987.

[Schueller 1997] G. I. Schueller, "A state-of-the-art report on computational stochastic mechanics", Probab. Eng. Mech. 12:4 (1997), 197-321.

[Schueller 2001] G. I. Schueller, “Computational stochastic mechanics: recent advances”, Comput. Struct. 79:22-25 (2001), 2225-2234.

[Sudret and der Kiureghian 2000] B. Sudret and A. der Kiureghian, "Stochastic finite element methods and reliability: a state-of-the-art report", Report No. UCB/SEMM-2000/08, Department of Civil and Environmental Engineering, University of California, Berkeley, November 2000, available at http://tinyurl.com/UCB-SEMM-2000-08.

[Ting 1996] T. C. T. Ting, Anisotropic elasticity: theory and applications, Oxford Engineering Science Series 45, Oxford University Press, New York, 1996.

Received 6 Apr 2010. Revised 5 Oct 2010. Accepted 12 Nov 2010.

SEVERINE LEPAGE: severine.lepage@e-xstream.com

Beckman Institute for Advanced Science and Technology, University of Illinois at Urbana-Champaign, 405 N. Mathews Av., Urbana, IL 61801, United States

Current address: Present address:, e-Xstream engineering (L) sarl, ZI Bommelscheuer, L-4940 Bascharage, Luxemburg

FERnANDo V. STUMP: fstump2@illinois.edu

Department of Mechanical Science and Engineering, University of Illinois at Urbana-Champaign, 1206 W. Green St., Urbana, IL 61801, United States

ISAIAH H. KIM: hdkim1@illinois .edu

Department of Mechanical Science and Engineering, University of Illinois at Urbana-Champaign, 1206 W. Green St., Urbana, IL, 61801, United States

Philippe H. Geubelle: geubelle@illinois.edu

Department of Aerospace Engineering, University of Illinois at Urbana-Champaign, 104 S. Wright St., Urbana, IL 61801, United States 


\title{
JOURNAL OF MECHANICS OF MATERIALS AND STRUCTURES
}

\author{
jomms.org
}

Founded by Charles R. Steele and Marie-Louise Steele

EDITORS

Charles R. SteEle

DAVIDE BIGONI

Stanford University, USA

YASUHIDE SHINDO

University of Illinois at Urbana-Champaign, USA

Tohoku University, Japan

\section{EDITORIAL BOARD}

$\begin{aligned} \text { H. D. BUI } & \text { École Polytechnique, France } \\ \text { J. P. CARTER } & \text { University of Sydney, Australia } \\ \text { R. M. CHRISTENSEN } & \text { Stanford University, USA } \\ \text { G. M. L. GLADWELL } & \text { University of Waterloo, Canada } \\ \text { D. H. HODGES } & \text { Georgia Institute of Technology, USA } \\ \text { J. HUTCHINSON } & \text { Harvard University, USA } \\ \text { C. HWU } & \text { National Cheng Kung University, Taiwan } \\ \text { B. L. KARIHALOO } & \text { University of Wales, UK } \\ \text { Y. Y. KIM } & \text { Seoul National University, Republic of Korea } \\ \text { Z. MROZ } & \text { Academy of Science, Poland } \\ \text { D. PAMPLONA } & \text { Universidade Católica do Rio de Janeiro, Brazil } \\ \text { M. B. RUBIN } & \text { Technion, Haifa, Israel } \\ \text { A. N. SHUPIKOV } & \text { Ukrainian Academy of Sciences, Ukraine } \\ \text { T. TARNAI } & \text { University Budapest, Hungary } \\ \text { F. Y. M. WAN } & \text { University of California, Irvine, USA } \\ \text { P. WRIGGERS } & \text { Universität Hannover, Germany } \\ \text { W. YANG } & \text { Tsinghua University, China } \\ \text { F. ZIEGLER } & \text { Technische Universität Wien, Austria } \\ & \\ \text { PRODUCTION } & \text { contact@ msp.org } \\ \text { SILVIO LEVY } & \text { Scientific Editor }\end{aligned}$

Cover design: Alex Scorpan

Cover photo: Mando Gomez, www.mandolux.com

See http://jomms.org for submission guidelines.

JoMMS (ISSN 1559-3959) is published in 10 issues a year. The subscription price for 2011 is US \$520/year for the electronic version, and \$690/year (+\$60 shipping outside the US) for print and electronic. Subscriptions, requests for back issues, and changes of address should be sent to Mathematical Sciences Publishers, Department of Mathematics, University of California, Berkeley, CA 94720-3840.

JoMMS peer-review and production is managed by EditFLow ${ }^{\circledR}$ from Mathematical Sciences Publishers.

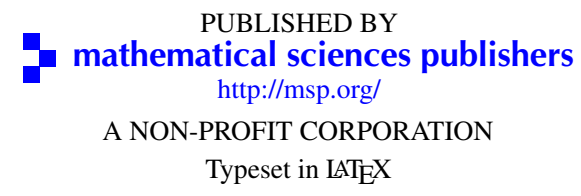

Copyright (C2011 by Mathematical Sciences Publishers 


\section{Journal of Mechanics of Materials and Structures}

\section{Volume 6, No. 7-8}

September-October 2011

\section{Special issue \\ Eleventh Pan-American Congress \\ of Applied Mechanics (PACAM XI)}

Preface

Adair R. Aguiar

949

Influence of specimen geometry on the Portevin-Le Châtelier effect due to dynamic strain aging

for the AA5083-H116 aluminum alloy

Rodrigo Nogueira de Codes and Ahmed Benallal

Dispersion relations for SH waves on a magnetoelectroelastic heterostructure with imperfect

interfaces

J. A. Otero, H. Calas, R. Rodríguez, J. Bravo, A. R. Aguiar and G. Monsivais

Numerical linear stability analysis of a thermocapillary-driven liquid bridge with magnetic stabilization

Yue Huang and Brent C. Houchens

Numerical investigation of director orientation and flow of nematic liquid crystals in a planar 1:4 expansion Pedro a. Cruz, Murilo F. Tomé, IAin W. Stewart and Sean McKee

Critical threshold and underlying dynamical phenomena in pedestrian-induced lateral vibrations of footbridges

Stefano LenCI and LAURA MARCHEgGiani

Free vibration of a simulation CANDU nuclear fuel bundle structure inside a tube

XUAN ZHANG and SHUdONG Yu

Nonlinear dynamics and sensitivity to imperfections in Augusti's model

D. Orlando, P. B. Gonçalves, G. Rega and S. LenCi

Active control of vortex-induced vibrations in offshore catenary risers: A nonlinear normal mode approach

CArlos E. N. MAZzilli and César T. SANCheS

Nonlinear electromechanical fields and localized polarization switching of piezoelectric macrofiber composites

Yasuhide Shindo, Fumio Narita, KoJi SATo and Tomo TAKeda

1089

Three-dimensional BEM analysis to assess delamination cracks between two transversely isotropic materials

Nicolás O. Larrosa, Jhonny E. Ortiz and Adrián P. Cisillino

Porcine dermis in uniaxial cyclic loading: Sample preparation, experimental results and modeling

A. E. Ehret, M. Hollenstein, E. MAzzA and M. Itskov

Analysis of nonstationary random processes using smooth decomposition

Rubens SAMpaio and Sergio Bellizzi

Perturbation stochastic finite element-based homogenization of polycrystalline materials

S. LePage, F. V. Stump, I. H. Kim and P. H. Geubelle

A collocation approach for spatial discretization of stochastic peridynamic modeling of fracture

Georgios I. Evangelatos and POL D. SPANOS 